\title{
Performance Of Modified Cosh Window Function
}

\author{
Shruti Jain \\ M.tech (E.C.E) \\ P.D.M. College of Engineering, Bahadurgarh, Haryana (India) \\ shrutier308@gmail.com ${ }^{1}$ \\ Dinesh Kumar Verma \\ Assistant Professor (E.C.E. Deptt.) \\ P.D.M. College of Engineering, Bahadurgarh, Haryana (India) \\ erdineshverma@gmail.com ${ }^{2}$
}

\begin{abstract}
In this paper, we have analyzed the performance of modified Cosh window function.A new parameter has been proposed to the previous 2-parameter Cosh window to improve its spectral characteristics in terms of ripple-ratio and transition width.It is observed by several iterations that by increasing the values of new parameter so proposed there is a significant variation in ripple ratio and transition width of the window spectrum.By the suitable combination of two parameters of the proposed window function, an optimum modified Cosh window is derived for $\mathrm{N}=51$ and $\mathrm{N}=101$. Optimum window provides better spectral characteristics. This modified window has applications in digital FIR designing, image processing as it provides better minimum stop band attenuation and contrast ratio respectively.An example has been taken to show the better performance of the proposed window for FIR filter designing for specified requirements.
\end{abstract}

Keywords: Cosh Window, Modified Cosh Window, Kaiser Window, Exponential Window, Ripple Ratio, FIR filters, Sidelobe Rolloff Ratio, Transition Width, Contrast ratio.

\section{Council for Innovative Research}

Peer Review Research Publishing System

Journal: INTERNATIONAL JOURNAL OF COMPUTERS \& TECHNOLOGY

Vol 9, No 3

editor@cirworld.com

www.cirworld.com, member.cirworld.com 


\section{INTRODUCTION}

Electronic filters are electronic circuits which perform signal processing functions, specifically to remove unwanted frequency components from the signal. Digital filters are classified as finite impulse response (FIR) and infinite impulse response (IIR) filters based on the duration of their impulse response. Window functions are widely used to truncate the infinite response of a filter to make the filter realizable. Various window functions have been proposed in literature.

Due to the flexible spectral properties of windows various windows like Dolph-Chebyshev, Kaiser, Barlett etc. have been proposed for signal processing applications. Since, the windows are sub-optimal solutions; every window is used according to the specifications required [13]. Cosh window when modified by introducing a new parameter, performs better in terms of ripple ratio. It provides better MSA as compared to Kaiser window, Cosh window, Exponential window in FIR filter designing [14].

\section{WINDOW DESIGN METHOD}

The desired frequency response of any digital filter is periodic in frequency and can be expanded in Fourier series[2,9], i.e.

$$
H_{d}\left(e^{j w}\right)=\sum_{n=-\infty}^{\infty} h_{d}(n) e^{-j w n}
$$

Where

$$
\begin{gathered}
h(n)=\quad \frac{1}{2 \pi} \int_{0}^{2 \pi} H\left(e^{j w}\right) e^{j w n} d w \\
h(n)=\frac{1}{2 \pi} \int_{-\pi}^{\pi} H(w) e^{j w n} d w \\
=\frac{1}{2 \pi} \int_{-w_{0}}^{w_{0}} e^{j w n} d w \\
=\frac{\sin \left(w_{0} n\right)}{\pi n}
\end{gathered}
$$

Window functions (or simply windows) are widely used in digital signal processing for the applications in signal analysis and estimation, digital filter design and speech processing. There are many windows that have been proposed and all are suboptimal solutions, the best window depends on the specific application.

\section{WINDOW FUNCTIONS}

Window functions (or simply windows) are widely used in digital signal processing for the applications in signal analysis and estimation, digital filter design and speech processing. There are many windows that have been proposed and all are suboptimal solutions, the best window depends on the specific application. The Key parameters of a window function are[2,3]:

1. Length of window function in time domain, $\mathrm{N}$.

2. Main lobe width of window's amplitude spectrum in frequency domain, D.

3. Ripple ratio and Side lobe roll-off ratio of windows spectrum in frequency domain, $R$ \& $S$ respectively

\subsection{COSH WINDOW}

The abbreviation COSH stands for Hyperbolic Cosine. A window based on this function is called as COSH window. The $\mathrm{COSH}$ Function and $\mathrm{COSH}$ window are given by following mathematical equations [10,12]:

$w[n]=\frac{\cosh \left(\alpha \sqrt{1-\left(\frac{2 n}{N-1}\right)^{2}}\right)}{\cosh (\alpha)} ; \quad 0 \leq n \leq N-1 \quad$ (5)

Here, $\mathrm{N}$ is the length of window in time domain, $\alpha$ is the adjustable window parameter.

\subsection{MODIFIED COSH WINDOW}

Modified Cosh window is simply a 3-parameter Cosh window. The basis function for this window is again Hyperbolic Cosine function. Mathematically, the modified Cosh window is given as[10,13,14]:

$w[n]=\left(\frac{\cosh \left(\alpha \sqrt{1-\left(\frac{2 n}{N-1}\right)^{2}}\right)}{\cosh (\alpha)}\right)^{\rho} ; 0 \leq n \leq N-1$

Where, $\mathrm{N}$ = length of window function

$\alpha, \rho=$ Shape changing parameters for window

Fig. 1[10] shows that Cosh window, Kaiser window, Exponential window and Modified Cosh window all have same shape characteristics. 


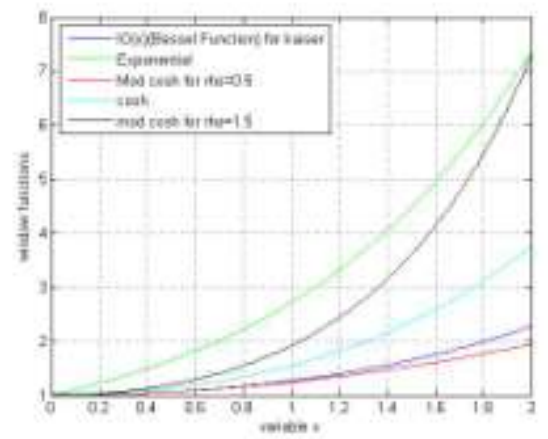

\section{Fig 1Similar Basis Functions of Several Windows}

The procedure to calculate the window's spectral parameters is show by fig. 2. A typical window is taken and its frequency spectrum is plotted. The dotted lines mark the values of spectral parameters in $\mathrm{db}$ and $\mathrm{rad} / \mathrm{sec}$ respectively.

Further the values are shown, how to observe and calculate them accurately.

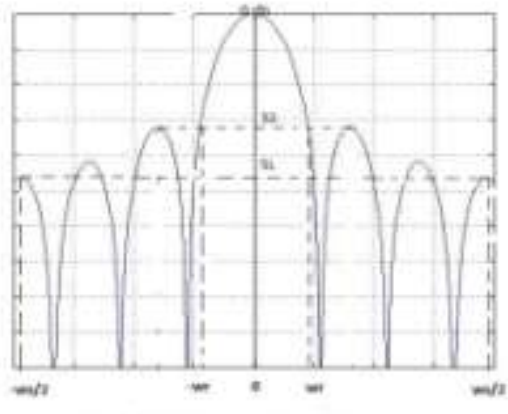

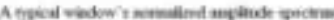

Fig. 2 Amplitude Spectrum for a general window

Form the above figure [10,14],

Ripple Ratio = Maximum side lobe amplitude in $\mathrm{dB}$ - Main lobe amplitude in $\mathrm{db}=\mathbf{S} \mathbf{d} \mathbf{d b}$.

Side lobe Roll off Ratio = Maximum side lobe amplitude in dB- Minimum side lobe amplitude in dB =S1 - SL db.

Main lobe width $=2 \mathrm{wr} \mathrm{rad} / \mathrm{sec}$.

Normalized Main lobe width i.e. $\mathrm{D}=2 \mathrm{wr}(\mathrm{N}-1) \mathrm{rad} / \mathrm{sec}[10]$.

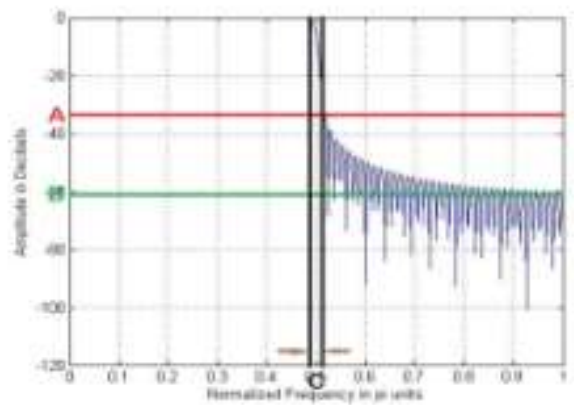

Fig 3 Frequency Response of an LPF Using Modified Cosh Window Function

From fig. 3, we can calculate the following parameters for a LPF[13]:

(i) Minimum Stop band Attenuation i.e. $\mathbf{M S A}=\mathbf{A} \mathbf{~ d b}$.

(ii) Far-end Stop band Attenuation i.e. FSA = B db.

(iii) Transition width i.e. $\Delta \mathbf{w}=\mathbf{C} \mathbf{d b}$.

All these parameters form the basis of comparison of low pass filters designed using different window functions [11].

Now, modified Cosh window function for different values of its third shaping parameter $\rho$, so introduced is plotted as shown in fig. 4 . 


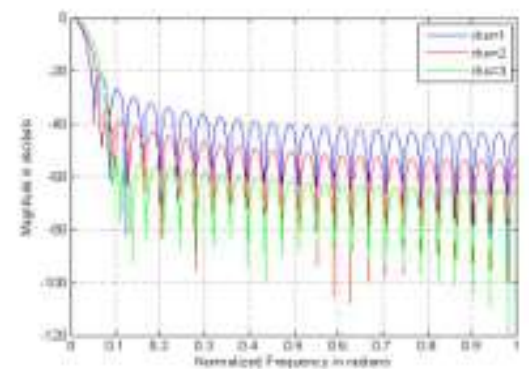

Fig. 4 Modified Cosh Window for Different $\rho$

Table $1 \mathrm{R}, \mathrm{S} \& \mathrm{D}$ for Modified Cosh Window for Constant $\alpha$ and Varying $\rho$

\begin{tabular}{|c|c|c|c|}
\hline S. No & $\begin{array}{c}\text { Ripple } \\
\text { Ratio, } \\
R(d b)\end{array}$ & $\begin{array}{c}\text { Sidelobe } \\
\text { Rolloff } \\
\text { Ratio, } \\
\text { S(db) }\end{array}$ & $\begin{array}{c}\text { Normalized } \\
\text { Width, } \\
\text { D(rad/sec) }\end{array}$ \\
\hline$\rho=1$ & 21.63 & 21.97 & 5.1 \\
\hline$\rho=2$ & 34.04 & 19.86 & 6.8 \\
\hline$\rho=3$ & 52.71 & 12.10 & 9.3 \\
\hline$\rho=4$ & 64.67 & 11.46 & 11.33 \\
\hline
\end{tabular}

From the above table, it is observed that as we keep on increasing the values of $\rho$, the ripple ratio and transition width increases and sidelobe roll-off ratio decreases as well respectively. So the parameter so proposed has a significant effect on all the three spectral properties of a window function.

\subsection{FIR FILTER DESIGNING}

Modified Cosh window provides better MSA for the LPF designing. It provides better FSA for same window length.

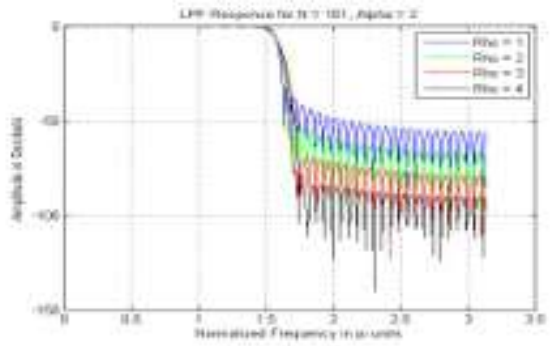

Fig. 5 LPF Designing Using Modified Cosh Window

\section{RESULTS \& DISCUSSIONS}

\subsection{RIPPLE RATIO VS. NORMALIZED WIDTH FOR DIFFERENT VALUES OF a AND $\rho$ FOR} $\mathrm{N}=51$

Ripple ratio vs. normalized width of different modified Cosh windows and basic Cosh window have been plotted. By keeping $\alpha$ constant and varying $\rho$, various values of ripple ratio and normalized width of the proposed window have been observed and plotted as shown in fig. 5.

At larger values of $\alpha$, Modified Cosh window behaves like a Cosh window. Modified Cosh window will perform better for some particular values of its parameters. It does not have power series expansion in its time domain function [2,12,14]. It performs better ripple ratio than 3-parameter ultra spherical window for wider main lobe width and larger side lobe roll-off ratio. Filters designed by Modified Cosh window provide better far end stop band attenuation than the filters designed by Kaiser Window. 


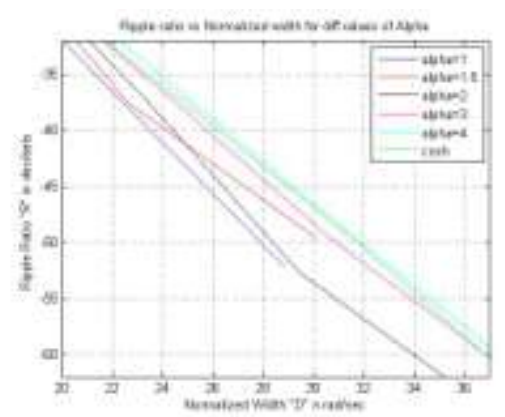

Fig.6 R vs. D for $\mathrm{N}=51$

Table 2 Data for Effect of $\rho$ on Modified Cosh Window

\begin{tabular}{|c|c|c|c|}
\hline Window & $\mathrm{N}$ & Amc & pmc \\
\hline Cosh & 51 & $0-9.5$ & $0-7.5$ \\
\hline Mod Cosh-1 & 51 & 1 & $0-3.4$ \\
\hline Mod Cosh-2 & 51 & 1.5 & $0-4.0$ \\
\hline Mod Cosh-3 & 51 & 3 & $1-3$ \\
\hline Mod Cosh-4 & 51 & 4 & $1-2.5$ \\
\hline Mod Cosh-5 & 51 & & \\
\hline
\end{tabular}

\subsection{RELATION BETWEEN FOR $\alpha$ AND $\rho \mathrm{N}=51 \& \mathrm{~N}=101$}

The relation between $\alpha$ and $\rho$ of modified Cosh window can be developed by keeping window size constant, then varying one of them and producing a table between these parameters and Ripple ratio. $R$ ranging from $-13.26 \leq R \leq-60 \mathrm{db}[12]$.

For $\mathrm{N}=51$

amc $=-5.832 \times 10^{-5} R^{8}-9.276 \times 10^{-3} R^{3}-0.531 R-8.446$

$\rho \mathrm{mc}=2.526 \times 10^{-5} R^{4}+5.065 \times 10^{-3} R^{3}+0.38 R^{2}+12.46 R+160.1$

(8)

For $\mathrm{N}=101$

$\alpha \mathrm{mc}=-5.098 \times 10^{-5} R^{5}-9.869 \times 10^{-3} R^{3}-0.564 R-9.027$

$\rho \mathrm{mc}=3.197 \times 10^{-5} R^{4}+6.353 \times 10^{-3} R^{3}+0.47 R^{2}+15.53 R+193.8$

A table is obtained for values of rho and alpha for a fixed window length and ripple ratio by several iterations. Values are obtained by varying them empirically. The design equations are formed by using Curve Fitting tool in MATLAB.

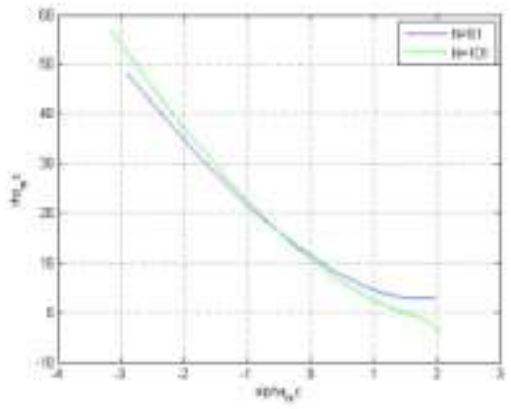

Fig. $7 \propto$ vs. $\rho$ for $N=51 \& N=101$

\subsection{OPTIMUM MODIFIED COSH WINDOW}

Now, from the above table, an optimum modified Cosh window is generated for $N=51$ \& $N=101$.Several readings are taken to plot the relationship between two windows 
$1^{\text {st }}$ window, $\mathrm{N}=51$

$2^{\text {nd }}$ Window, $\mathrm{N}=101$

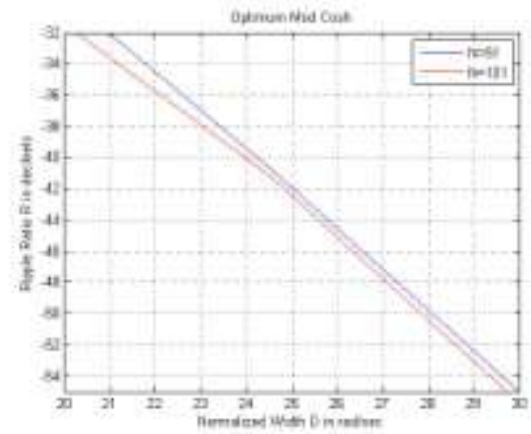

Fig. 8 R vs. D For optimum modified Cosh window

From the plot, we found

Optimum Modified Cosh window for different window sizes, $\mathrm{N}$ and fixed $\mathrm{R}$ and wr. It can be seen that on increasing the window size i.e. $\mathrm{N}$ the ripple ratio increases for the same transition width and same parameters. It is found that Optimum proposed window occurs at the parameters where their combinations provide equal amplitude of side-lobes including the first one adjacent to the main lobe. This also shows that on increasing the window size ripple ratio also increases.

\subsection{COMPARISON OF COSH, MODIFIED COSH, KAISER \& EXPONENTIAL WINDOWS}

Frequency domain spectrums of Cosh, Kaiser, Exponential and Modified Cosh Windows are drawn on the same ground for fixed values of ripple ratio, $\mathrm{R}$ and Normalized transition width, $\mathrm{D}$.

$R=-60 \mathrm{db}$.

$D=0.158 \mathrm{rad} / \mathrm{sec}$

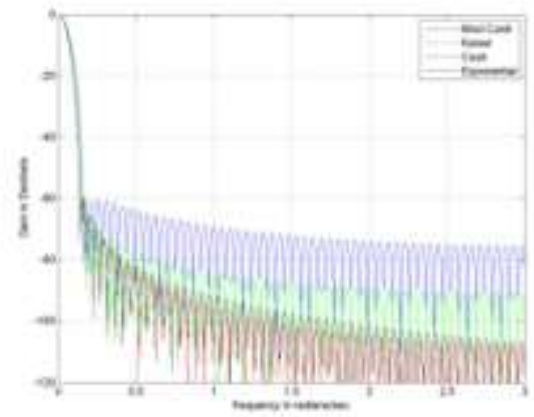

Fig.9 Optimum Modified Cosh, Cosh, Kaiser\& Exponential windows in frequency domain

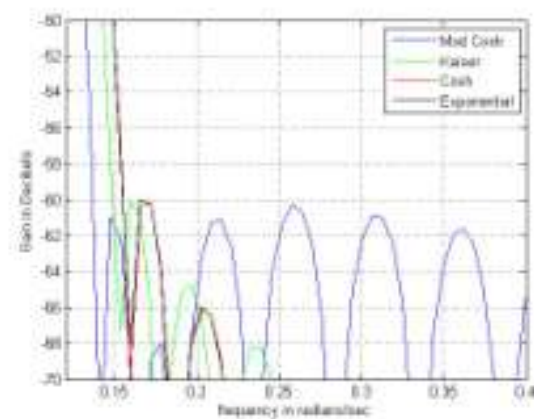

Fig. 10 Zoom in view of Fig. 7 
Table 3 Mod Cosh, Kaiser\& Cosh window

\begin{tabular}{|c|c|c|c|c|c|}
\hline Window & $\begin{array}{c}\mathrm{R} \\
(-\mathrm{db})\end{array}$ & $\begin{array}{c}\text { wr } \\
(\mathrm{rad} / \mathrm{sec})\end{array}$ & $\rho$ & $\alpha$ & $\mathrm{N}$ \\
\hline $\begin{array}{c}\text { Modified } \\
\text { Cosh }\end{array}$ & 60 & .158 & 3.3 & 2.05 & 101 \\
\hline Kaiser & 60 & .158 & - & 8.2 & 109 \\
\hline Cosh & 60 & .159 & 1 & 8.3 & 115 \\
\hline Exp & 60 & .159 & - & 8.3 & 117 \\
\hline
\end{tabular}

Modified Cosh window provides better ripple ratio as compared to other windows for the same window length. Modified Cosh window and other windows ripple ratio difference decreases as window length $\mathrm{N}$ increases. It can be concluded that Modified Cosh window approaches 2-parameter Cosh window as window length increases.

\subsection{COMPARISON IN APPLICATION TO FIR FILTER DESIGNING}

Let us consider a design example:

Cut-off frequency, $\omega c=0.5 \mathrm{rad} / \mathrm{sec}=[(\mathrm{wp}+\mathrm{ws}) / 2]$

Pass band frequency, $\omega p=0.45 \mathrm{rad} / \mathrm{sec}$

Stop-band Frequency, $\omega \mathrm{s}=0.55 \mathrm{rad} / \mathrm{sec}$

Window length, $\mathrm{N}=101$

Transition Width, wr $=0.171 \mathrm{rad} / \mathrm{sec}$

Parameters for Different Windows:

Table 4 Parameters Choices

\begin{tabular}{|c|c|c|}
\hline Window Used & $\alpha$ & $\rho$ \\
\hline Kaiser & 6 & - \\
\hline Cosh & 6 & - \\
\hline Exponential & 6 & - \\
\hline Modified Cosh & 3.74 & 1.815 \\
\hline
\end{tabular}

The following plots have been recovered by using the above requirements for designing of a LPF and specifications for the window functions.

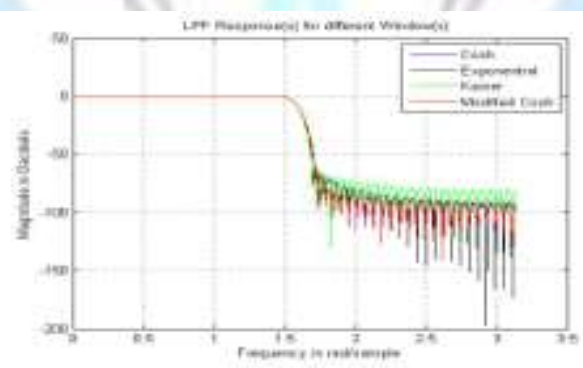

Fig. 11 Amplitude Spectrum of LPF designed by using different windows

By above fig., we analyze following points:

Modified Cosh Window provides highest MSA than all other windows. FSA of Modified Cosh Window is the second highest. Exponential Window provides highest FSA compared to all other windows.

Table 5 MSA and FSA for Different Windows

\begin{tabular}{|c|c|c|}
\hline Window Used & MSA (rad/sec) & FSA (rad/sec) \\
\hline Kaiser & 63.09 & 81.22 \\
\hline Cosh & 61.61 & 91.31 \\
\hline Exponential & 61.65 & 93.74 \\
\hline Modified Cosh & 68.11 & 93.09 \\
\hline
\end{tabular}




\section{CONCLUSION}

The covered work was done on studying and analyzing the proposed window performance on varying its parameters. It is found that an increase in the new parameter results in wider main-lobe width and smaller ripple ratio. The performance is counted on the basis of its spectral characteristics which are Ripple ratio, Side-lobe Roll-off ratio and Transition Width. In the result and discussion part, various plots are discussed related to modified Cosh window function in time and frequency domain and its dependence on parameters. Its performance is compared with Cosh, Exponential and Kaiser Windows with respect to spectral parameters.Specific values of proposed window parameters which are responsible for changing its shape and characteristics are derived for an optimum modified Cosh window. The results of window length comparison with Kaiser, Exponential and Cosh windows, it is observed that the minimum window length required for satisfying a given main-lobe width and ripple ratio is obtained by the proposed modified Cosh window.Optimum Modified Cosh window provides better ripple ratio for same window length. LPF designed by Modified Cosh Window provides highest MSA and second highest FSA than all other windows considered. Modified Cosh window provides better side lobe roll-off characteristics then Kaiser Window, and the difference becomes larger as the normalized main lobe width increases. Kaiser window performs slightly better than modified Cosh window, in terms of ripple ratio and the difference becomes larger as the normalized main lobe width increases.

\section{REFERENCES}

[I] T. S. E1-A1i, Discrete Systems and Digital Signal Processing with MATLAB, CRC Press, 2004

[2] A. Antoniou, Digital Signal Processing: Signal, Systems, and Filters, McGraw-Hili, 2005

[3] S. W. A. Bergen and A. Antoniou, "Design of Ultraspherical window functions with prescribed spectral characteristics" EURASIP Journal on Applied Signal ProceSSing, no.I3, 2004, pp. 2053-2065

[4] F.Kaiser and R.W.Schafer, "On the use of the lo-sinh window for spectrum analysis" IEEE Trans. AcoustiCS, Speech, and Signal Processing, vol.28, no.I, 1980, pp.105-107

[5] F. Kaiser, "Nonrecursive digital filter design using IO-sinh window function" in Proc. IEEE Int. Symp. Circuits and Systems (ISCAS74), San Francisco, Calif, USA, 1974, pp.20-23

[6] S. W. A. Bergen and A. Antoniou, "Design of Nonrecursive Digital Filters Using the Ultraspherical Window Function" EURASIP Journal on Applied Signal ProceSSing, no.I2, 2005, pp. 1910- 1922

[7] A. Jain, R. Saxena, S.C. Saxena, "A simple alias-free QMF system with near-perfect reconstruction" J.indian Ins.Sci., Jan-Feb, no.12, 2005, pp. 1-10

[8] C. L. Dolph, "A Current Distribution for Broadside Arrays Which Optimizes the Relationship Between Beamwidth and Side-lobe Level" Proc.

IRE, vo1.34, pp.335-348, June 1946.

[9] T. Saramaki, "A class of window functions with nearly minimum sidelobe energy for designing FIR filters" in Proc. IEEE Int. Symp. Circuits and systems (ISCAS'89), Portland, Ore, USA, yoU, pp. 359 - 362, 1989.

[10] K. Avci, A. Nacaroglu, "Cosine hyperbolic window family with its application to FIR filter design". Proceedings of 3rd International Conference

on Information \& Communication Technologies: from Theory to Application (ICTIA'08), pp. 289-290, Damascus, Syria, April 2008.

[II] K. Avci, Arif Nacaroglu," A New Window Based on Exponential Function". Proceedings of 3rd International Conference on Information \& Communication Technologies: from Theory to Applications (ICTTA'08), pp. 289-290, Damascus, Syria, April 2008.

[12] K. Avci, N. Nakaroglu, " High Quality Low Order Nonrecursive Digital Filter Design Using Modified Kaiser Window", IEEE international Conference ,2008.

[13] Kemal Avci and Arif Nacaroglu, "Modification of Cosh window Family", ICTTA, 2008.

[14] Pankaj Shukla, Vikas Soni and Mithilesh Kumar," Non recursive digital FIR Filter design by 3-parameter Hyperbolic Cosine Window: A High Quality Low order Filter Design “, IEEE, 2011. 